\title{
Systematic Observation of Time-Dependent Phenomena in the RF Output Spectrum of High Power Gyrotrons
}

\author{
Andreas Schlaich ${ }^{1,2, a}$, Gerd Gantenbein ${ }^{2}$, Stefan Kern ${ }^{2}$, and Manfred Thumm ${ }^{1,2}$ \\ 1 Karlsruhe Institute of Technology (KIT): Institut für Hochfrequenztechnik und Elektronik (IHE), \\ Engesserstraße 5, 76131 Karlsruhe, Germany \\ 2 Karlsruhe Institute of Technology (KIT): Institute for Pulsed Power and Microwave Technology (IHM), \\ Hermann-von-Helmholtz-Platz 1, 76344 Eggenstein-Leopoldshafen, Germany
}

\begin{abstract}
At IHM/KIT, high power gyrotrons with conventional cavity (e.g. $1 \mathrm{MW}$ $\mathrm{CW}$ at $140 \mathrm{GHz}$ for the stellarator Wendelstein $7-\mathrm{X}$ ) and coaxial cavity (2 MW shortpulse at $170 \mathrm{GHz}$ for ITER) for fusion applications are being developed and verified experimentally. Especially with respect to the problem of parasitic RF oscillations in the beam tunnel of some W7-X tubes, investigations of the gyrotron RF output spectrum have proved to be a valuable source of diagnostic information. Signs of transient effects in millisecond pulses, like frequency switching or intermittent low-frequency modulation, have indicated that truly time-dependent measurements with high frequency resolution and dynamic range could give deeper insight into these phenomena. In this paper, an improved measurement system is presented, which employs a fast oscilloscope as receiver. Shorttime Fourier transform (STFT) is applied to the time-domain signal, yielding time-variant spectra with frequency resolutions only limited by acquisition length and STFT segmentation choice. Typical reasonable resolutions are in the range of $100 \mathrm{kHz}$ to $10 \mathrm{MHz}$ with a currently memory-limited maximum acquisition length of $4 \mathrm{~ms}$. A key feature of the system consists in the unambiguity of frequency measurement: The system receives through two parallel channels, each using a harmonic mixer $(h=9-12)$ to convert the signal from RF millimeter wave frequencies (full D-Band, $110-170 \mathrm{GHz})$ to IF $(0-3 \mathrm{GHz})$. For each IF output signal of each individual mixer, injection side and receiving harmonic are initially not known. Using accordingly determined LO frequencies, this information is retrieved from the redundancy of the channels, yielding unambiguously reconstructed $\mathrm{RF}$ spectra with a total span of twice the usable receiver IF bandwidth, up to $\approx 6 \mathrm{GHz}$ in our case. Using the system, which is still being improved continuously, various transient effects like cavity mode switching, parasitic oscillation frequency variation, and lowfrequency modulation have been documented.
\end{abstract}

\section{Introduction}

Fusion experiments throughout the world rely on a set of plasma heating methods, one of which is electron cyclotron resonance heating $(\mathrm{ECRH})$. High power continuous wave $(\mathrm{CW})$ electromagnetic waves are directed to a reactor chamber, where resonant coupling causes the carried power to be absorbed in the plasma in order to reach the temperatures necessary for a fusion reaction. For major reactors like Wendelstein 7-X (W7-X) or ITER, the coupling frequencies of interest for heating and additional tasks like electron cyclotron current drive (ECCD) are situated in the range of $100-170 \mathrm{GHz}$ with multi-megawatt $\mathrm{CW}$ power requirements, thus leaving only gyrotrons as realistic power sources[1].

At IHM/KIT, gyrotrons for both the W7-X stellarator and ITER are designed and tested [2].

\footnotetext{
a e-mail: andreas.schlaich@kit.edu
} 


\subsection{Gyrotrons for Fusion Science}

Modern high-power gyrotrons are vacuum tube diode or triode devices of about $3 \mathrm{~m}$ length. Typical parameters and a schematic axial cut can be found in figure 1.

DC power is supplied through the cathode, which contains an emitter ring creating an annular electron beam through temperature-limited thermal emission. The electrons are accelerated by the voltage $U_{\mathrm{k}}$ between cathode and anode to travel towards the collector, guided through the tube by a high axial magnetic flux density caused by a superconducting (SC) magnet. They finally hit the collector, which is either at anode potential or negative with respect to the anode for efficiency enhancement (“depressed collector operation").

The magnetic field furthermore effects the electrons to gyrate around their motion guiding center, such that in the resonator a significant part of their kinetic energy is available in a rotation in the plane transversal to the tube axis. In the gyrotron interaction, this transversal electron energy excites a high-order $\mathrm{TE}_{m, n}$ circular waveguide mode in the cylindrical resonator ("cavity").

The TE mode propagates from the resonator to a quasi-optical mode converter ("launcher"), which transforms the TE mode into a $\mathrm{TEM}_{00}$ wave. This Gaussian beam is directed by internal metallic mirrors, to be then leaving the gyrotron body transversally through a dielectric window, from where it is guided either by quasi-optical transmission lines or corrugated waveguides to the reactor chamber.

\subsection{KIT/IHM High Power Teststand}

In most gyrotron testing experiments at IHM, the tube is operated in short-pulse mode (SP), i.e. repetitive pulses of duration $T_{\mathrm{p}}=0.5-5 \mathrm{~ms}$. Only when a gyrotron performs well in SP operation, longpulse (LP) operation of several $100 \mathrm{~ms}$ up to 30 minutes ("CW") can be safely considered.

Monitoring $U_{\mathrm{k}}, I_{\mathrm{b}}$ and measuring the output power using a calorimetric load gives basic data on the gyrotron's performance, however more information can be obtained from the RF output spectrum.

\begin{tabular}{ll}
\hline Parameter & Value \\
\hline Cavity frequency & $140 \mathrm{GHz}$ \\
CW RF output power & $1 \mathrm{MW}$ \\
Cavity type & conventional \\
Cavity mode & $\mathrm{TE}_{28,8}$ \\
Cathode voltage & $80 \mathrm{kV}$ \\
Beam current & $40 \mathrm{~A}$ \\
Peak magnetic flux density & $5.57 \mathrm{~T}$ \\
Efficiency (depressed collector) & $45 \%$ \\
\hline
\end{tabular}

(a) Performance parameters of W7-X gyrotrons

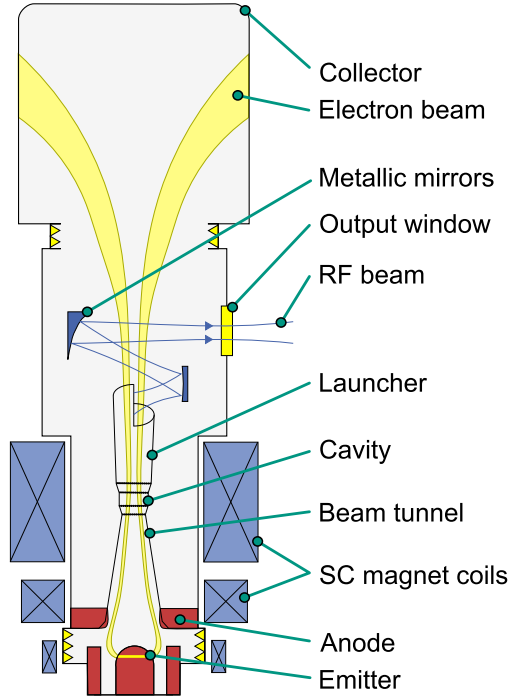

(b) Schematic axial cut of a modern high-power diode type gyrotron including superconducting (SC) magnet

Fig. 1: Gyrotron overview information 
Primary monitoring system at IHM is a filterbank, which supplies 8 channels of $2 \mathrm{GHz}$ width each, applicable in the 110-170 GHz (D) band with a dynamic range of $24 \mathrm{~dB}$. Its main purpose is giving a spectral overview and providing existence information on the cavity mode, competitors and significant parasitic oscillations.

For more detailed examination of the dominant oscillation's behaviour over time, a frequencytime-analyzer with $2 \mathrm{GHz}$ bandwidth can be employed, but is less sensitive than the filterbank branch. This combined system is described in [3].

The measurement signal is typically picked up with an overmoded waveguide from stray radiation emitted by the gyrotron's main output window or relief windows. Since the original frequency range of interest is the D-Band, downmixing to an intermediate frequency (IF) of several GHz is necessary for signal acquisition. The harmonic mixers employed for this are optimized for minimum conversion loss on one specified mixing harmonic ( $h_{\mathrm{S}}=9$ for the systems described above), but since frequency mixing is achieved through the nonlinearity of one or several diodes, other harmonics $h_{\mathrm{S}} \pm i$ will convert signals from radio frequency (RF, here $110-170 \mathrm{GHz}$ ) to IF, too. At an IF receiver, this creates a frequency ambiguity since the receiving harmonic is unknown. Another source of ambiguities is the lack of knowledge about injection side $^{1}$ of the mixing process.

Although sequential measurements on several pulses without changing gyrotron parameters can resolve the aforementioned frequency ambiguities, this is a crude method and relies on exact spectral reproducability of output spectra in repeated pulses.

\subsection{Improvement Goals}

The importance of high-dynamic range spectrum monitoring and exact as well as unambiguous frequency determination has been established during measurement campaigns starting in 2009, a process which was accompanied by the development of a spectrum analyzer based measurement system. Here, parasitic oscillations in the beam tunnel of W7-X gyrotron SN4 were diagnosed, located and removed by a dedicated design change [4].

Repeated observation of spurious parasitic oscillations categorized after cavity interaction (ACI, see [5], [6], [7]) in various gyrotrons since 2010/2011, which exhibited low reproducability between pulses due to frequency variance during pulses. This observation shaped the development goals for an extension of the existing gyrotron monitoring systems:

- Dynamic range should be at least comparable to that of the existing spectrum analyzer based system (effectively $50 \mathrm{~dB}$, see [8]).

- Broad spectral coverage: At least one complete filterbank channel (2 GHz) should be covered.

- Time variance information as accurate as possible is highly desirable.

- Unambiguous frequency information with at least $10 \mathrm{MHz}$ resolution should be provided.

\section{New Pulse Analysis System}

A new pulse analysis system to meet the goals stated in the previous section has been developed and applied at the IHM gyrotron test stand in a prototype state during winter 2011/12.

In the following section, the hardware setup is presented and explained, followed by more detailed explanations and remarks about the subsequent steps of the signal processing chain and the underlying concept of the system (see also figure 2).

\subsection{RF Frontend}

After a level control system for mixer protection from RF overload, the signal is split and directed to the harmonic mixers. Here the transition from RF (WR6 waveguides) to IF (SMA coaxial) takes place. Maximum input power for the mixers is $0 \mathrm{dBm}$, while conversion losses are approximately $27-35 \mathrm{~dB}$.

\footnotetext{
1 A normal mixer can receive through the upper side band $\left(h f_{\mathrm{LO}}<f_{\mathrm{RF}}\right.$, low side injection) and through the lower side band $\left(h f_{\mathrm{LO}}>f_{\mathrm{RF}}\right.$, high side injection $)$.
} 


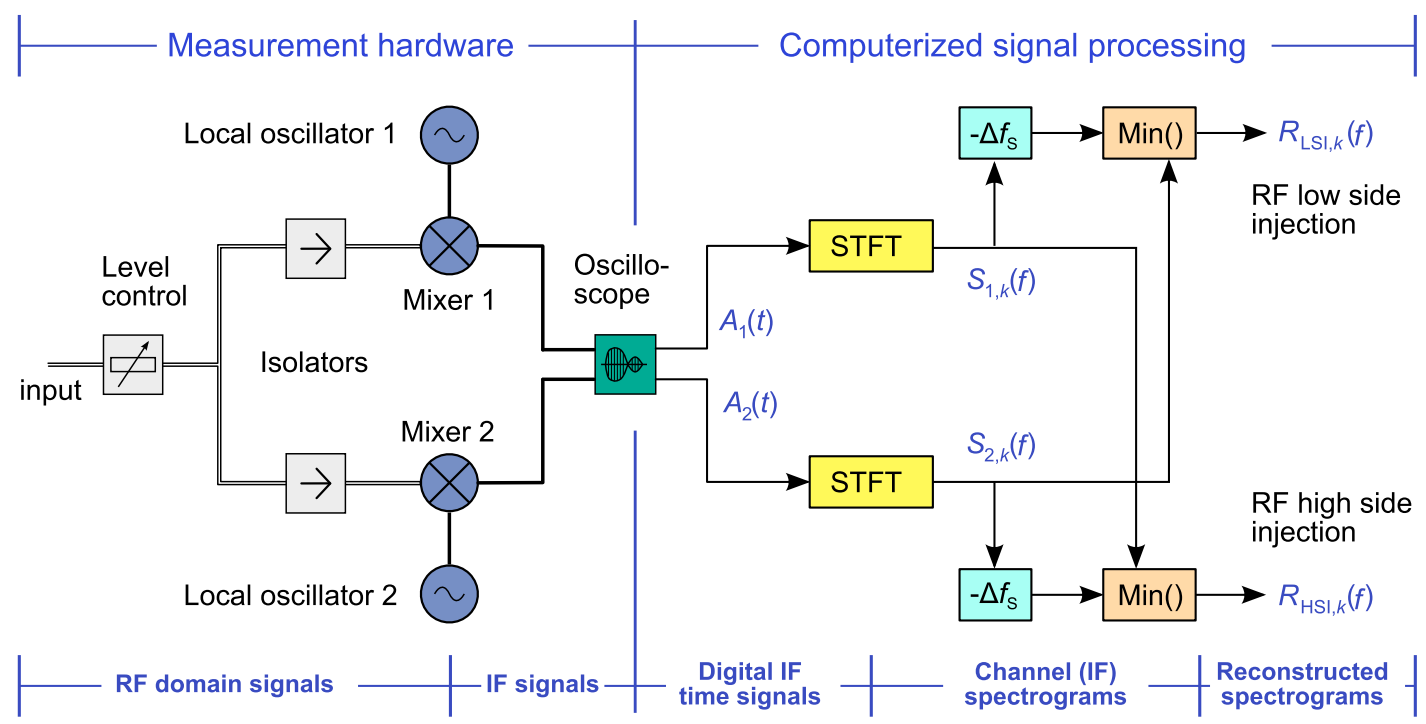

Fig. 2: System setup and data flow in new pulse analysis system

\subsection{Acquisition}

After downconversion, signals are detected using a fast oscilloscope, whose parameters and settings determine most of the basic properties of the system. The 8 bit analog-digital converter's (ADC) sampling rate $(\mathrm{SR})$ of $f_{\mathrm{ADC}}=10 \mathrm{GS} / \mathrm{s}$ allows aliasing-free input signals at maximum frequencies identical to the Nyquist frequency of $f_{\mathrm{N}}=5 \mathrm{GHz}$. With the currently installed memory of $80 \mathrm{MS}$ shared between the two receiving channels this allows for a maximum gapless acquisition length of $4 \mathrm{~ms}$, which can cover regular experimental SP operation $\left(T_{\mathrm{p}}=1 . .5 \mathrm{~ms}\right)$ sufficiently.

\subsubsection{Dynamic Range}

With some safety margin, maximum power at the oscilloscope input due to mixing conversion loss can be expected to be $-35 \mathrm{dBm}$. At $50 \Omega$ impedance, this corresponds to a maximum input voltage of $\approx 4 \mathrm{mV}$, and with a minimum vertical range of $5 \mathrm{mV}$ the oscilloscope is able to employ its complete dynamic range.

Since the signals are analyzed in frequency domain, the effective dynamic range is strongly influenced by the FFT resolution. Following [9], signal-to-noise-ratio (SNR) can be estimated to be

$$
\mathrm{SNR} \approx 6.02 N_{\mathrm{ADC}}+1.76 \mathrm{~dB}+10 \log _{10} \frac{N_{\mathrm{S}}}{2} \approx 82.1 \mathrm{~dB} .^{2}
$$

\subsubsection{Bandwidth}

As necessary for all ADCs, a low pass filter is included in the oscilloscope's data acquisition path for aliasing suppression. Generally, without low-pass filtering, a signal of frequency $f>f_{\mathrm{N}}$ will appear to the receiver at frequencies $f_{\mathrm{IF}}=\left|2\left\lfloor\frac{f+f_{\mathrm{N}}}{2 f_{\mathrm{N}}}\right\rfloor f_{\mathrm{N}}-f\right|{ }^{3}$ With a non-ideal low pass filter, obviously the range $f_{\mathrm{N}}<f<2 f_{\mathrm{N}}$ holds the highest risk of not fully suppressed aliasing products.

${ }^{2}$ Calculated using a resolution of $\delta f_{\mathrm{Num}}=1 \mathrm{MHz}$ and the specified ADC effective number of bits (ENOB) of $N_{\mathrm{ADC}}=7.2$. The sensitivity cannot be directly estimated this way, since LO noise from the mixing process will form the lower boundary of detectable power.

3 The expression $[\cdot\rfloor$ here denotes the floor()-function, i.e. next lower integer of the argument. 
Since high dynamic range for the system is intended, remaining aliasing products must be taken into account. Figure 3 contains the relative received power for a synthesizer signal in the frequency range up to $2 f_{\mathrm{N}}$ directly applied to the scope. ("scope LP"). Without additional measures for aliasing suppression, the aliasing free dynamic range (AFDR) would be less than $30 \mathrm{~dB}$.

External IF low pass filters are not necessary though, since the mixers employed as receiver contain a diplexer unit for separation of LO and IF. These have LP characteristic in the IF path, which has also to be taken into account when assessing aliasing limits. The "diplexer LP" curves in figure 3 show the diplexer IF response as measured with a network analyzer. The low pass effect of the two superposed responses ("Combination")) is strong enough to ensure an AFDR of $>60 \mathrm{~dB}$ with a span $S=3 \mathrm{GHz}$. The receiver sensitivity drops by $10 \mathrm{~dB}$ between $\mathrm{DC}$ and $f_{\mathrm{IF}}=S$, which is still acceptable for a system whose primary purpose is frequency determination. However care is necessary when questions about presence or absence of signals are risen, since a weak signal at the edge of the chosen span could be overlooked.

\subsection{Transistion to Frequency Domain}

\subsubsection{Basics on Short-time Fourier Transform}

Since information on time variance is desired, the short time fourier transform (STFT) is applied to each sampled IF time signal $A_{i}(t)$ ( $i=1,2$ denotes channel indices). This means dividing each $A_{i}(t)$ into multiple segments $a_{i, k}(t)$ of identical duration $T_{\text {seg. }}$. From each time segment, one spectrum $s_{i, k}(f)$ with a minimum frequency discretization $\delta f_{\text {Num }}=\frac{1}{T_{\text {seg }}}$ is created by FFT, where the index $k$ indicates the time position.

E.g. for a time signal $A_{i}(t)$ of duration $T_{\mathrm{A}}=4 \mathrm{~ms}$, demanding $\delta f_{\mathrm{Num}}=1 \mathrm{MHz}$ results for each $i$ in 4000 segments $a_{i, k}(t)(1<k \leq 4000)$ with $T_{\text {seg }}=\frac{1}{f_{\mathrm{Num}}}=1 \mu \mathrm{s}$. Fourier transform $a_{i, k}(t) \circ \longrightarrow s_{i, k}(f)$ yields $i \cdot k=8000$ spectra, while within the fourier time-frequency uncertainty, each spectrum $s_{i, k}$ is associated with the time location of its signal segment $a_{i, k}$ in the underlying original time signal of channel $i$. Aligning $s_{i, k}(f)$ along $k$ forms a $2 \mathrm{D}$ spectrogram ${ }^{5} S_{i, k}(f)$ of $A_{i}(t)$.

\footnotetext{
4 It has to be noted that the oscilloscope is only vendor-specified for a maximum span of $2 \mathrm{GHz}$ because of this low pass characteristic curve.

5 In this publication, the frequency axis is horizontal and the time axis advances from bottom to top of each plot, while intensity is given in $\mathrm{dBm}$. Depending on the application, there exist spectrogram implementations with alternative axes alignment and power scaling.
}

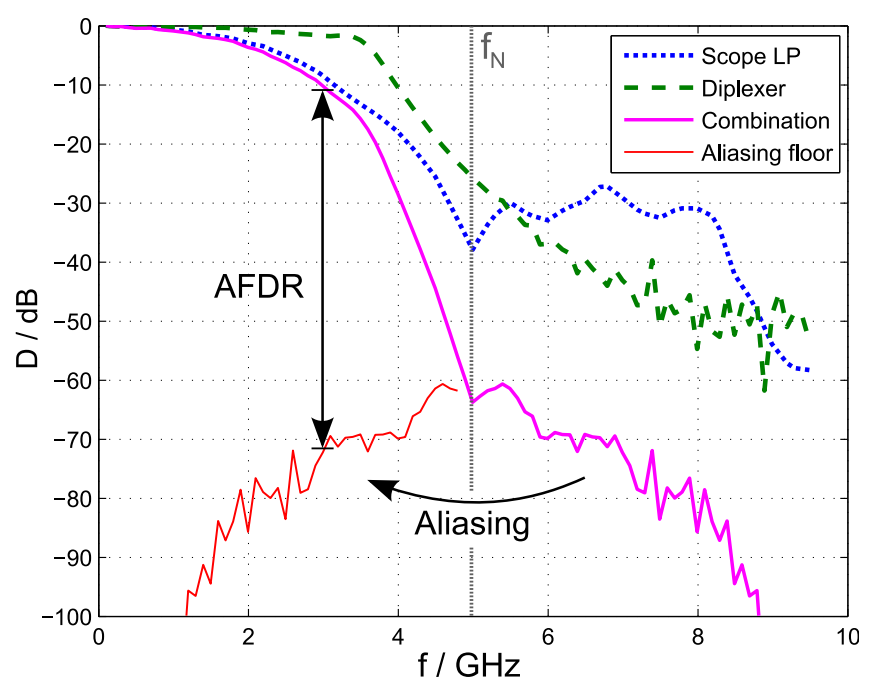

Fig. 3. Directly measured input low-pass of oscilloscope ("Scope LP"), diplexer transmission ("Diplexer LP") and combined frequency response ("Combination"). For a sampling rate of $10 \mathrm{GS} / \mathrm{s}$, aliasing starts at $f_{\mathrm{N}}=5 \mathrm{GHz}$. Signals in $f_{\mathrm{N}}<f<2 f_{\mathrm{N}}$ will be visible at $f_{\mathrm{IF}}=10 \mathrm{GHz}-f$, as indicated in the graph ("Aliasing floor", see also section 2.2.2). By setting the maximum sensitivity loss for maximum input frequency and the desired AFDR, the usable IF frequency bandwidth $B_{\mathrm{IF}}$, or span, can be determined. In this case, for AFDR = $60 \mathrm{~dB}, B_{\mathrm{IF}}=3 \mathrm{GHz}$ is attained. Sensitivity however suffers $10 \mathrm{~dB}$ reduction towards the edge due to the oscilloscope low pass filter. 


\subsubsection{Overlap Factor}

In the described application of STFT, chosing $\delta f_{\text {Num }}$ automatically fixes the reciprocally defined spectrogram time step. This relation can be superficially decoupled by allowing the time signal segments to overlap, hence creating a further degree of freedom with the overlap factor $x_{\mathrm{OV}}=[0 . .1)$ which allows to increase the number of spectra. $x_{\mathrm{OV}}=0$ would create disjunct time segments as in the above STFT description, while for $x_{\mathrm{OV}}>0$ increasing segment overlap achieved (see figure 4 and annotation).

Reasonable overlap values range between 0.1 and 0.9 , and often $x_{\mathrm{OV}}=0.5$ is chosen to have every segment halfway covered by its neighbours. It has to be noted that though using $x_{\mathrm{OV}}>0$ smoothes the display in time direction and provides smaller time steps, time resolution itself remains unchanged.

\subsubsection{Choice of Window Function}

While frequency resolution is important for the system described, sidelobe suppression is paramount to maximize the ability of the system to detect weak signals in the presence of strong nearby signals. The minimum 3-Term Blackman-Harris window was chosen, which yields a frequency resolution augmentation factor just under 2 over the theoretical optimum of the rectangular window $\left(\rightarrow \delta f_{\text {eff }} \approx\right.$ $\left.2 \delta f_{\text {Num }}\right)$, and ensures $-67 \mathrm{~dB}$ sidelobe suppression with low scalloping loss and fast side lobe roll-off. ${ }^{6}$

Since FFT window functions are tapered to equal zero on their edges, $A_{i}(t)$ samples at the center of STFT segments $a_{i, k}(t)$ contribute stronger to the resulting spectrogram than parts at the edges, which are attenuated by the window function. This is a strong argument for an overlapping factor $x_{\mathrm{OV}}$ choice larger than zero, since otherwise signal variations in order of $T_{\text {seg }}$ can be lost.

\subsection{RF Spectrogram Reconstruction}

\subsubsection{Ambiguity Sources and Compensation}

The $\mathrm{RF}$ reconstruction process creates unambiguous $\mathrm{RF}$ spectrograms $R_{\mathrm{HSI}, k}(f), R_{\mathrm{LSI}, k}(f)$ from the previously calculated, and still ambiguous, IF spectrograms $S_{i, k}(f)$. Here the configuration of the local oscillators used for the initial downconversion from RF to IF becomes relevant.

Let $f_{\mathrm{LO}}$ be the local oscillator frequency, through which in combination with the utilized mixer harmonic $h$ the RF frequency $f_{\mathrm{RF}}$ is received in IF as

$$
f_{\mathrm{IF}}=\left|h f_{\mathrm{LO}}-f_{\mathrm{RF}}\right| .
$$

Considering $N$ possible harmonics $h$ for an arbitrary IF signal with unknown injection side yields $2 N$-fold frequency ambiguity through this relationship. This ambiguity can be resolved by exploiting the data from two parallel channels and limiting the parameters of "valid" reception to a predefined combination of $h_{\mathrm{S}, 1}$ and $h_{\mathrm{S}, 2}$. All signals which are not received in channel $i$ through $h_{\mathrm{S}, i}$ are invalid and to be discarded.

${ }^{6}$ A great overview of FFT window effects and selection criteria can be found in [10].

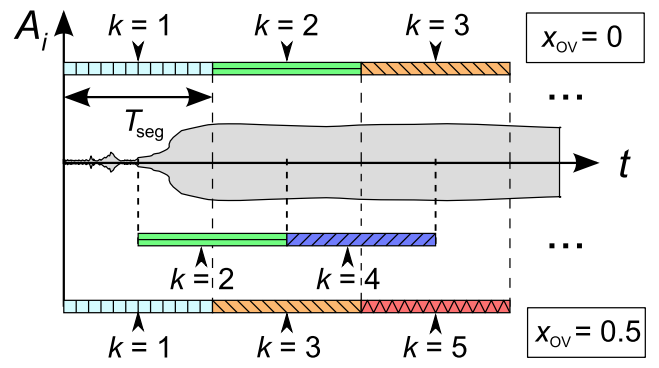

Fig. 4. STFT segmentation of time signal $A_{i}(t)$ into time signal segments $a_{i, 1}(t), a_{i, 2}(t), \ldots, a_{i, k}(t)$. For $x_{\mathrm{OV}}=0$ (top), segments are directly adjacent and non-overlapping in time, so the spectra calculated from each segment will be uncorrelated. For STFT segmentation with $x_{\mathrm{OV}}=0.5$ (bottom), overlapping segments are created. Even though a spectrogram based on the resulting segments will have more time steps, time resolution will be the same as for $x_{\mathrm{OV}}=0$; also the power in frequency points of adjacent spectra will be correlated in time direction. 
To enable this decision, a fixed relation between "valid" IF signals in both channels must be established. The definition

$$
\Delta f_{\mathrm{S}}=h_{1, \mathrm{~S}} \cdot f_{\mathrm{LO}, 1}-h_{2, \mathrm{~S}} \cdot f_{\mathrm{LO}, 2}
$$

yields an IF link that satisfies the previous requirement: For each signal in channel 1 at frequency $f_{\mathrm{IF}, 1}$ to be considered valid (i.e. received through the pre-defined harmonics $h_{i, \mathrm{~S}}$ ) a corresponding signal in channel 2 at frequency $f_{\mathrm{IF}, 2}=f_{\mathrm{IF}, 1} \pm \Delta f_{\mathrm{S}}$ must be found. Since $h_{i, \mathrm{~S}}$ are known a priori, true RF frequencies can be recalculated from the measured IF frequencies for valid signals.

By evaluating the direction of frequency offset (i.e. the sign of $f_{\mathrm{IF}, 1}-f_{\mathrm{IF}, 2}=\Delta f_{\mathrm{IF}}$ ) with (3) as

$$
h_{1, \mathrm{~S}} \cdot f_{\mathrm{LO}, 1}>h_{2, \mathrm{~S}} \cdot f_{\mathrm{LO}, 2} \Rightarrow \Delta f_{\mathrm{S}}>0 \Leftrightarrow \begin{cases}f_{\mathrm{IF}, 1}<f_{\mathrm{IF}, 2} \Rightarrow \Delta f_{\mathrm{IF}}<0 & \text {, low side injection } \\ f_{\mathrm{IF}, 1}>f_{\mathrm{IF}, 2} \Rightarrow \Delta f_{\mathrm{IF}}>0 & \text {, high side injection }\end{cases}
$$

the injection side ambiguity can be solved in the same step. ${ }^{7}$

\subsubsection{Unambiguity Confidence Aspects}

Only signals with a bandwidth smaller than $\Delta f_{\mathrm{S}}$ can be unambiguously reconstructed. For normal gyrotron operation, $\Delta f_{\mathrm{S}}<100 \mathrm{MHz}$ suffices. However on the occurrence of broadband signals, $\Delta f_{\mathrm{S}}$ must be adjusted.

The confidence of unambiguity against signals received through harmonics $h_{i, n}=h_{i, \mathrm{~S}} \pm l_{i, n}$ is dependent on $h_{i, \mathrm{~S}}$, for which two cases can be separated:

- For systems with with $h_{1, \mathrm{~S}}=h_{2, \mathrm{~S}}$, most dangerous are signals received over $h_{i, \mathrm{~S}} \pm 1$. These create $\Delta f_{\mathrm{IF}, x}=-\Delta f_{\mathrm{S}}\left(1 \pm \frac{1}{h_{n, \mathrm{~S}}}\right)$. Even if the receiver can resolve $\left|\Delta f_{\mathrm{IF}, x}\right|-\left|\Delta f_{\mathrm{S}}\right|$, it still has to be made sure that the original signals themselves are narrowband. E.g. for $h_{i, \mathrm{~S}}=12$ and $\Delta f_{\mathrm{S}}=65 \mathrm{MHz}$, ambiguity difference is ||$\Delta f_{x}|-| \Delta f_{\mathrm{S}} \| \approx 5.4 \mathrm{MHz}$, which is a rather strict constraint.

- If $h_{1, \mathrm{~S}} \neq h_{1, \mathrm{~S}}$, reception through wrong harmonics can only produce $\Delta f_{\mathrm{X}} \approx f_{\mathrm{RF}}\left(\frac{l_{1, n}}{h_{1, \mathrm{~S}}}-\frac{l_{2, n}}{h_{2, \mathrm{~S}}}\right) \gg \Delta f_{\mathrm{S}}$, so for these configurations confidence is much larger.

From the considerations in section 2.2.2, aliasing signals will appear "mirrored" at $f_{\mathrm{N}}$. For the RF reconstruction this would result in $\Delta f_{\mathrm{IF}, x}=\Delta f_{\mathrm{S}}$ and therefore misinterpretation as correct harmonic, but switched injection side. For this reason, aliasing signals lead to flawed reconstruction, cannot be detected by the system and have to be prevented by design.

\subsubsection{Implemented Reconstruction Technique}

The described frequency validation is to be applied to every spectral line pair $\left[s_{1, k}(f), s_{2, k}(f)\right]$, and is obviously independent of the time axis. Furthermore, along the $f$ axis, it can be implemented frequency point-based, it is straightforward to use a low-level correlation approach for implementation.

To ensure high non-linearity of the operation in combination with simplicity and intuitiveness as far as possible, especially still the possibility of result formulation in physical quantities familiar to RF engineering, the Minimum()-Operation was chosen as correlation metric. With the previous definitions, $\mathrm{RF}$ spectrogram reconstruction can be formulated as

$$
\begin{array}{ll}
R_{\mathrm{LSI}, k}=\operatorname{Min}\left(S_{1, k}(f), S_{2, k}(f-\Delta f)\right) & \forall k \\
R_{\mathrm{HSI}, k}=\operatorname{Min}\left(S_{1, k}(f-\Delta f), S_{2, k}(f)\right) & \forall k .
\end{array}
$$

As a result, the reconstructed spectra retain the power unit of the input spectra and no additional scaling factors, detection/confidence coefficients are necessary. The result from this operation, as indicated in fig 2, are reconstructed RF spectrograms. Since the original spectrograms $S_{i}$ are shifted by $\Delta f_{\mathrm{S}}$ vice versa in the process, between $R_{\mathrm{HSI}}$ and $R_{\mathrm{LSI}}$ a gap ("blind spot") of width $\Delta f_{\mathrm{S}}$ is inevitable.

High- and low side injection are evaluated equally, so with an IF detection bandwidth $B_{\mathrm{IF}}$ the resulting real time bandwidth of the system is $2 B_{\mathrm{IF}}-\Delta f_{\mathrm{S}}$.

\footnotetext{
${ }^{7}$ Definition (3) could also be formed inversely, leading to a switch of signs in (4) but no further consequences.
} 


\section{Measurements}

In the following, various transient phenomena which were observed with the system during prototype status measurements with several W7-X gyrotrons are presented. ${ }^{8}$ The nature of the effects is explained in brief, however no deeper scientific assessment of the physical backgrounds is pursued within this publication.

The measurements were accompanied by continuous monitoring of $U_{\mathrm{k}}$ and $I_{\mathrm{b}}$ using midrange oscilloscopes ( $\mathrm{SR}<1 \mathrm{GS} / \mathrm{s}$ ), yet this data is omitted here for brevity.

Since for typical settings one measurement result consists of spectrograms extending several thousand pixels along both time and frequency axis, full reproduction in print would be compromised. For this reason, in the following instead of complete reconstructed spectrograms only crops (zoomed areas of interest) will be used.

\subsection{Cavity Mode Switch}

Operation at a cavity mode's edge of stability parameters can cause it to destabilize or become subject to competition with neighbouring modes.

In figure 5, the end of a short pulse operated with the nominal W7-X cavity mode of $\mathrm{TE}_{28,8}$ is visible. Until $T=1.924 \mathrm{~ms}$ pulse time, $\mathrm{TE}_{28,8}$ operates stable at $f=140.26 \mathrm{GHz}$. Then mode competition with $\mathrm{TE}_{27,8}$ around $137.45 \mathrm{GHz}$ cause both modes to undergo multiple frequency jumps and intensity variation. Intermittent modulation side bands with $\approx 10 \mathrm{MHz}$ distance to both main lines are discernible.

Surprisingly, the mode competition interval leads to parallel and stable operation of both modes with dominating $\mathrm{TE}_{27,8}$ for $30 \mu$ s till the pulse is terminated by regular operating voltage shut down.

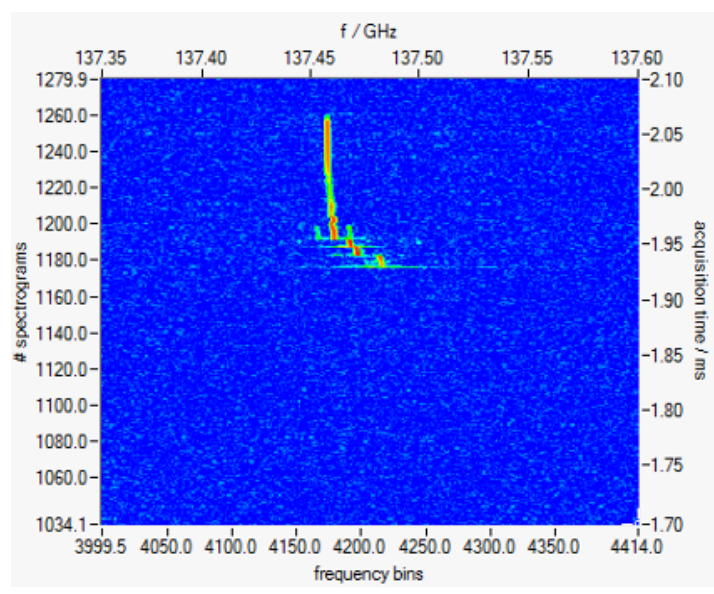

(a) HSI crop

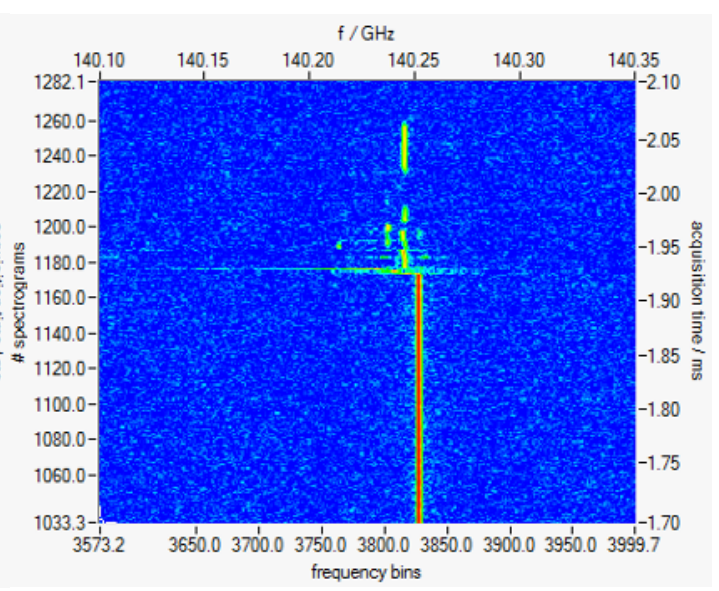

(b) LSI crop

Fig. 5: Simultaneuosly acquired spectrograms in HSI (a) and LSI (b) $\left(\delta f_{\text {Num }}=1 \mathrm{MHz}, x_{\mathrm{OV}}=0.25\right)$. Crops were made from spectrograms of originally $135-141 \mathrm{GHz}$ band width, with $\Delta f_{\mathrm{S}}=65 \mathrm{MHz}$. A cavity mode switch from $\mathrm{TE}_{28,8}(140.26 \mathrm{GHz})$ to $\mathrm{TE}_{27,8}(137.46 \mathrm{GHz})$ at pulse time $T=1.93 \mathrm{~ms}$ followed by frequency instabilities has been observed.

\footnotetext{
${ }^{8}$ For maximum unambiguity, mixers with $h_{1, \mathrm{~s}}=9$ and $h_{2, \mathrm{~s}}=12$ were chosen.
} 


\subsection{Low-Frequency Modulation}

In many occasions, especially for parasitic oscillations and dominant operation of a "wrong" cavity mode, low-frequency modulation side bands can be observed. Sideband distance and therefore modulation frequencies have been observed up to several hundred $\mathrm{MHz}$, however typical frequencies range between 3 and $50 \mathrm{MHz}$. In figure 6a, $750 \mu$ s stretch from a short pulse with continuous $\mathrm{TE}_{27,8}$ mode activity at $137.535 \mathrm{GHz}$ (a) and corresponding spectra at two time positions (b) is exemplified. Strong modulation sidebands, possibly caused by automodulation due to high reflection of this mode at the $\mathrm{RF}$ window, of discrete but alternating frequency dominate the spectrum.

\subsection{Parasitic Frequency Jumping}

Tests of W7-X gyrotron SN6 yielded similar behaviour as previous examinations of SN4R, reliable operation of about $900 \mathrm{~kW}$. For some operation points, especially for beam currents above $44 \mathrm{~A}$, parasitic oscillations in the lower $130 \mathrm{GHz}$ could be detected. Figure 7 shows an excerpt from the trace of a typical specimen.

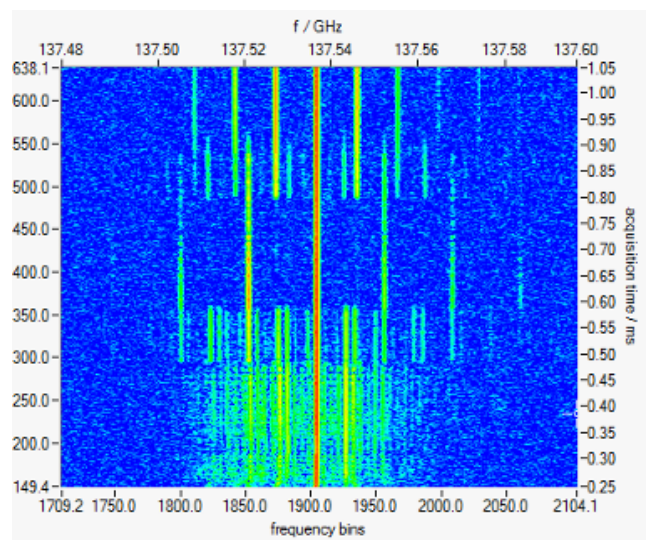

(a) Spectrogram

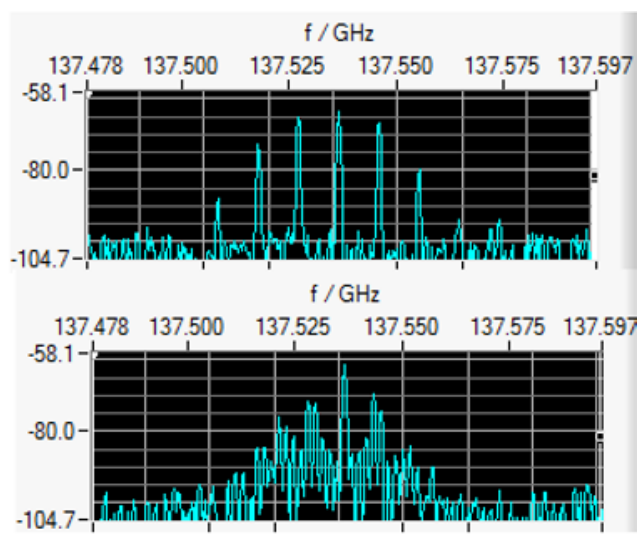

(b) Spectra at $T=1.0 \mathrm{~ms}$ (upper), $T=0.40 \mathrm{~ms}$ (lower)

Fig. 6: Alternating LF modulation frequencies in a pulse with stable $\mathrm{TE}_{27,8}$ mode activity.

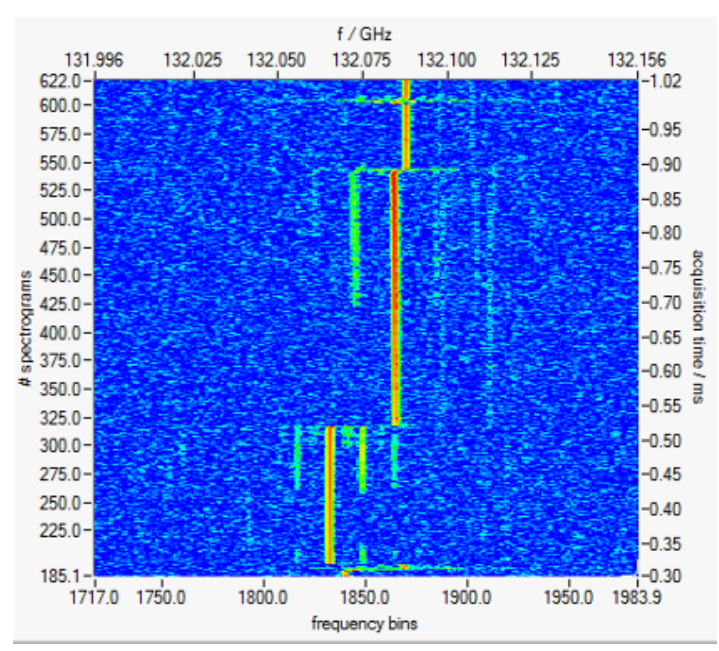

Fig. 7. Measurement of parasitic oscillation, supposedly dynamic ACI [6], in W7-X gyrotron SN6 during factory acceptance test preparations in early 2012 . Jumping between discrete frequencies accompanied by spectral distortions like low-frequency modulation and broad-band signatures is obvious. This observation strongly corroborates hypotheses on discrete parasitic frequency jumping formulated earlier [5]. 


\section{Conclusion}

\subsection{Summary and Outlook}

The problem of time-variant phenomema in gyrotron output spectra lead to the development of a timebased spectrum analysis system, which can create high-resolution spectrograms of complete short pulses with a real time bandwidth of currently $6 \mathrm{GHz}$. Already during the first experimental operation, a novel quality of gyrotron output spectrum data could be gathered, which is yet to be fully exploited.

The system is still under development and has not reached its final hardware configuration yet. Optimization techniques as mixer conversion loss minimization through LO power adaption have yet to be employed. A thorough full band characterization of the system is already planned.

Also the control and analysis software is under continuous improvement, and still has much potential for speed gain and feature extension.

Furthermore, an extension of the system has been decided, which will add a second receiver channel pair to the system. Then arbitrary events can be monitored in real-time, even if the spectral distance exceeds the receiver's IF bandwidth by far. Especially simultaneuos documentation of cavity oscillation behaviour in the context of parasitic oscillations, where the latter can appear more than $10 \mathrm{GHz}$ apart from the former, will be easily possible.

The authors would like to thank Tomasz Rzesnicki, Andrey Samartsev and John Jelonnek (IHM), and François Legrand, Gérald Lietaer (TED) for their kind support.

This work, supported by the European Communities under the contract of Association between EURATOM and KIT, was carried out within the framework of the European Fusion Development Agreement. The views and opinions expressed herein do not necessarily reflect those of the European Commission.

\section{References}

1. M. Thumm, State-of-the-Art of High Power Gyro-Devices and Free-Electron Masers - Update 2011, KIT Scientific Reports 7606, (2012)

2. M. Thumm, Progress on Gyrotrons for ITER and Future Thermonuclear Fusion Reactors, IEEE Transactions on Plasma Science vol. 39, issue 4, (2011)

3. O. Prinz, Aufbau eines breitbandigen Echtzeit-Frequenzmesssystems für mm-Wellen Hochleistungsgyrotrons, Forschungszentrum Karlsruhe - Wissenschaftliche Berichte FZKA7020, 2004

4. G. Gantenbein et al., Experimental Investigations and Analysis of Parasitic RF Oscillations in High-Power Gyrotrons, IEEE Transactions on Plasma Science vol. 38, issue 6, (2010)

5. A. Schlaich et al., Examination of Parasitic After-Cavity Oscillations in the W7-X Series Gyrotron $S N 4 R$, 36th International Conference on Infrared, Millimeter and Terahertz Waves (IRMMW-THz), 10.1109/irmmw-THz.2011.6104902, 2011

6. S. Kern et al., Simulation and Experimental Investigations on Dynamic After Cavity Interaction (ACI), 35th International Conference on Infrared, Millimeter and Terahertz Waves (IRMMW-THz), 10.1109/ICIMW.2010.5612609, 2010

7. S. Alberti et al., Gyrotron Parasitic-Effects Studies using the Time-Dependent Self-Consistent Monomode Code TWANG, 36th International Conference on Infrared, Millimeter and Terahertz Waves (IRMMW-THz), 10.1109/irmmw-THz.2011.6105097, 2011

8. A. Schlaich, Aufbau und Anwendung eines Systems zur Spektralanalyse von Gyrotronpulsen im Millimeterwellenbereich, KIT Scientific Reports 7541, (2010)

9. J. G. Proakis, Digital Signal Processing : Principles, Algorithms, and Applications (Pearson Prentice Hall, NJ/US 2007)

10. F. J. Harris, On the Use of Windows for Harmonic Analysis with the Discrete Fourier Transform, Proceedings of the IEEE 66 No.1, (1978) pp. 51-83 